\title{
Experiências de Criação de Aplicativos Móveis com Alunos do Ensino Fundamental e Médio
}

\author{
Ana Luísa V. Solórzano ${ }^{1}$, Andrea S. Charão ${ }^{2}$ \\ Patrícia P. Barcelos ${ }^{2}$ \\ ${ }^{1}$ Curso de Ciência da Computação \\ ${ }^{2}$ Departamento de Linguagens e Sistemas de Computação \\ Universidade Federal de Santa Maria \\ Santa Maria, RS, Brasil \\ \{alsolorzano, andrea, pitthan\}@inf.ufsm.br
}

\begin{abstract}
Resumo. A disseminação de fundamentos de programação a crianças e jovens é alvo de diversas iniciativas atualmente, inspiradas em seus potenciais benefícios para esta geração. Neste trabalho, apresenta-se as experiências de um projeto de extensão universitária que incentivou alunos de Ensino Fundamental e Médio a produzirem aplicativos móveis. No projeto, utilizou-se a ferramenta MIT App Inventor como introdução à programação, através de encontros semanais para a produção de um aplicativo de perguntas e respostas. A atividade colocou em evidência obstáculos a serem resolvidos, como maneiras de melhor adequar a didática utilizada e de manter os encontros atrativos. De forma geral, as experiências mostraram-se estimulantes tanto para o público-alvo como para os graduandos que se envolveram no projeto.
\end{abstract}

\begin{abstract}
Disseminating programming fundamentals to children and youth is the goal of several initiatives inspired by its potential benefits for this generation. In this work, we present the experience of a university extension project that encouraged students of primary and secondary education to create mobile applications. In the project, we used the MIT App Inventor tool as a programming introduction, through weekly meetings to produce a questions and answers mobile application. The activity has highlighted obstacles to be overcome, such as ways to better tailor the didactic used and to keep the meetings attractive. Overall, the experience proved to be stimulating both for the audience and for undergraduate students who were involved in the project.
\end{abstract}

\section{Introdução}

Atualmente, pode-se observar uma forte dependência de jovens com tecnologias móveis [Rideout et al. 2010]. Por serem de fácil acesso e simples uso, os aplicativos (apps) para dispositivos móveis atingiram grande popularidade, intermediando tarefas úteis em áreas variadas como segurança, educação e lazer. Dados publicados em 2015 apontam que, no mundo todo, o número de "viciados em usar aplicativos", isto é, pessoas que os utilizam pelo menos 60 vezes ao dia, é maior do que a população do Brasil [Khalaf 2015].

De fato, a atual geração de jovens espera que existam aplicativos (apps) para tudo e muitas vezes recusa formas menos diretas e eficientes de obterem o que desejam, sendo 
considerados por estudiosos como a "Geração App" [Gardner e Davis 2013]. Por outro lado, os benefícios dos apps são relevantes, pois eles podem promover um senso de identidade, manter amizades e estimular a criatividade [Gardner e Davis 2013].

$\mathrm{Na}$ área de educação, há algum tempo vislumbra-se que aplicativos móveis podem ser aliados para estimular o aprendizado [Hulme e Traxler 2005]. Mais do que isso, na educação em Computação, acredita-se que a criação de aplicativos móveis possa ser uma forma de incentivar as novas gerações a desenvolver o pensamento computacional [Wolber 2011, Fronza et al. 2015].

Assim, no contexto de um projeto de extensão universitária, desenvolveu-se uma experiência de criação de aplicativos com alunos de Ensino Fundamental e Médio, despertando-os para a ideia de que eles estão aptos a não somente utilizar, mas também a produzir essas tecnologias. Para isso, utilizou-se a ferramenta MIT App Inventor, que propõe uma abordagem lúdica e auto-explicativa, usando programação visual com blocos que se encaixam. Apesar da plataforma apresentar apenas recursos para criação de aplicativos simples, estima-se que ela também sirva de incentivo para futuros programadores, pois pode despertar a busca por funcionalidades mais avançadas fazendo uso de outras linguagens de programação.

Neste artigo, relata-se a experiência de um ano de exploração do MIT App Inventor no cenário delineado acima. A Seção 2 descreve a ferramenta trabalhada e suas potencialidades. A Seção 3 apresenta trabalhos que também utilizam esta ferramenta, mas com algumas diferenças de abordagem. A Seção 4 apresenta o método de trabalho e as atividades realizadas nas experiências. A seção 5 apresenta e discute resultados de avaliação das experiências, ressaltando alguns obstáculos identificados. Por fim, a Seção 6 traz considerações finais, destacando lições aprendidas.

\section{MIT App Inventor}

O MIT App Inventor é um ambiente web lançado em 2010 pela empresa Google em parceria com o Instituto de Tecnologia de Massachusetts (MIT). Essa ferramenta é utilizada para criar aplicativos funcionais em dispositivos móveis de sistema operacional Android, possibilitando que pessoas sem conhecimento sobre linguagens de programação possam desenvolver aplicativos através de uma abordagem visual, com encaixe de blocos.

O ambiente de criação dos aplicativos é dividido em tela de "Design" e tela de "Blocos", para adicionar elementos visuais e lógicos, respectivamente. Dentre os elementos visuais (Figura 1) estão desde funcionalidades simples como inserir botões e imagens até mais elaboradas como localização, sensores e chamadas para celular.

Na tela de elementos lógicos (Figura 2), estão os blocos responsáveis pelo funcionamento do aplicativo, identificados por cor e formato e separados em blocos lógicos, de controle, textuais, aritméticos, para a criação listas, dentre outros.

O App Inventor disponibiliza tutoriais para iniciantes aprenderem o básico da programação com blocos e um emulador, visto que nem todos possuem um aparelho de celular. Além disso, o usuário pode publicar aplicativos na chamada "Galeria", facilitando o compartilhamento de suas criações e a descoberta de outras possibilidades com a ferramenta. 


\section{Trabalhos Relacionados}

A disseminação da lógica computacional a crianças e adolescentes é alvo de iniciativas em escala internacional, como por exemplo Hour of Code (Hora do Código) ${ }^{1}$ ou Europe Code Week ${ }^{2}$. Essas iniciativas multiplicam experiências bem sucedidas, como é o caso da programação visual por meio de blocos que se encaixam, presente não só no MIT App Inventor mas também na ferramenta precursora Scratch ou na mais recente Blockly. Dado o caráter inovador e o potencial dessas ferramentas, elas também têm sido alvo de trabalhos científicos e acadêmicos.

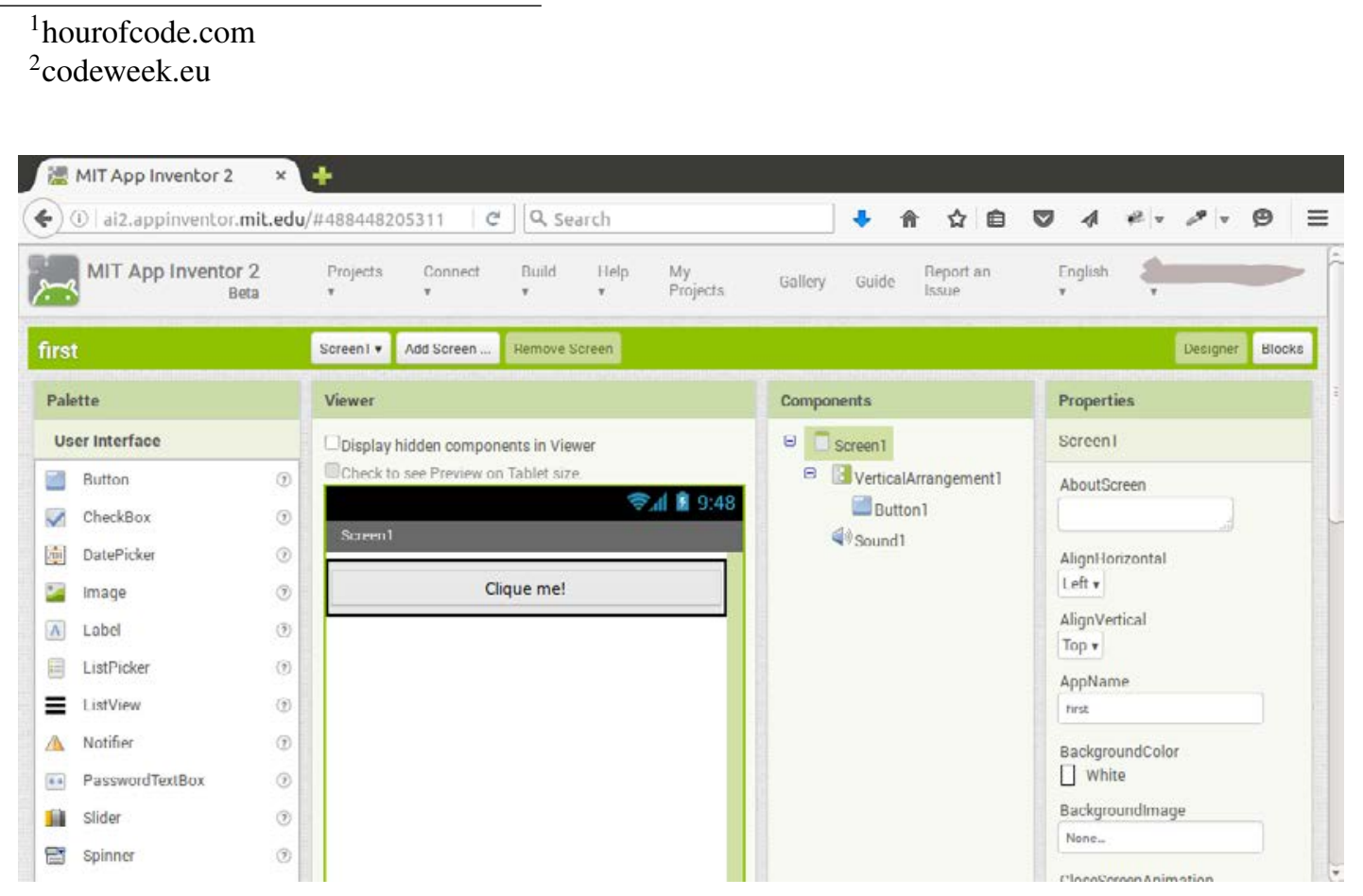

Figura 1. Tela "Design" do MIT App Inventor

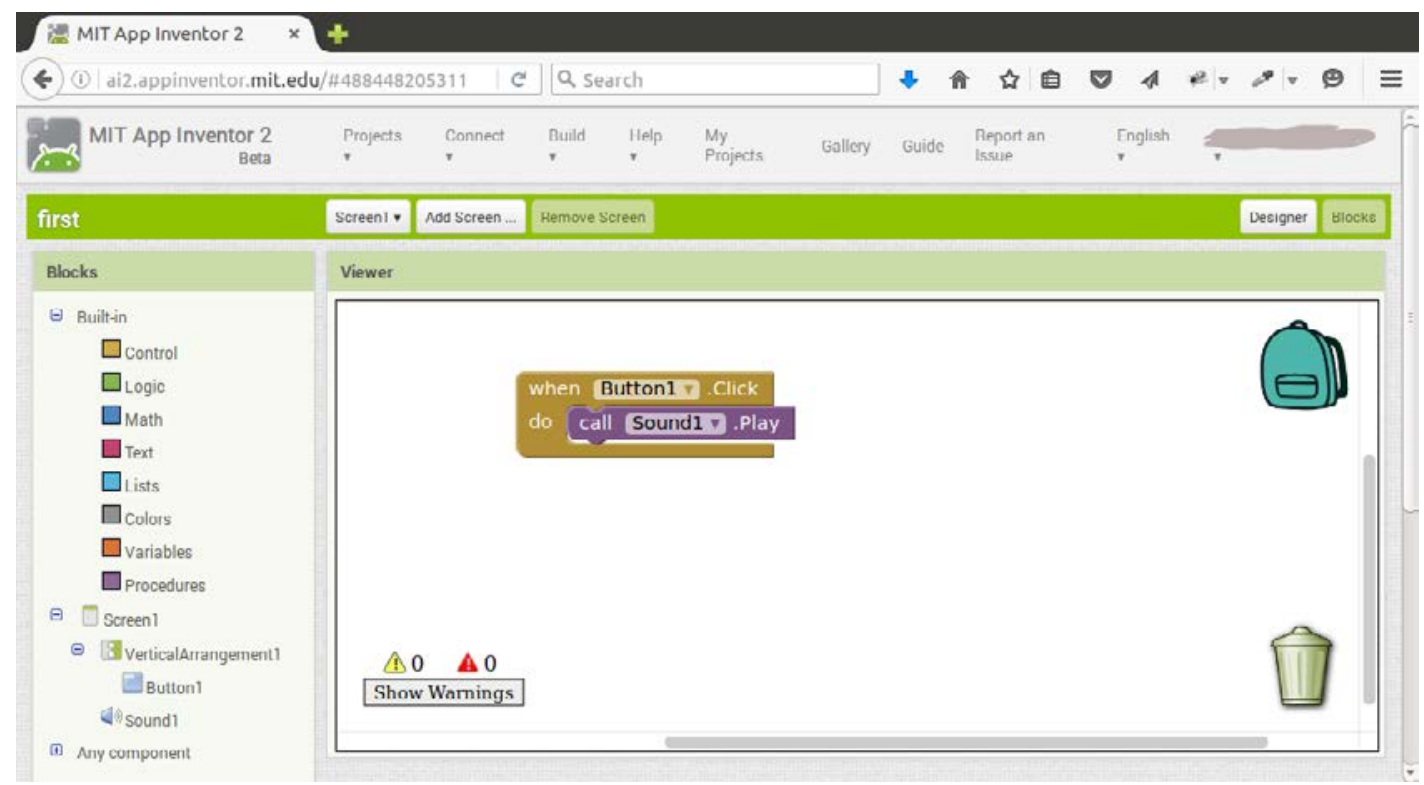

Figura 2. Tela "Blocos" do MIT App Inventor 
No Brasil, encontram-se relatos de experiência de uso do App Inventor em projetos de instituições de ensino. Por exemplo, há trabalhos que relatam a utilização da ferramenta para ensino de lógica e programação no ensino médio e também a calouros no ensino superior [Gomes e de Melo 2013a, Gomes e de Melo 2013b, Finizola et al. 2014, de Cerqueira Cardoso et al. 2015]. Já em [Duda et al. 2014], a ferramenta é usada por bolsistas de cursos técnicos, para a criação de jogos digitais envolvendo tópicos em matemática. Em [Ramos et al. 2015], o App Inventor é utilizado em um projeto visando estimular alunas de ensino médio a considerarem carreiras em ciências exatas, engenharias e, especialmente, computação.

Nota-se, nos trabalhos citados, que os autores se preocupam em ressaltar aspectos positivos das experiências. Apenas no trabalho de [Ramos et al. 2015] são comentadas dificuldades, algumas relacionadas à ferramenta e outras ao contexto no qual ela se inseriu. Ao nosso entendimento, para facilitar a reprodução das experiências em outros tempos e espaços, é importante que os relatos discutam os obstáculos que, naturalmente, podem ocorrer nesse tipo de experiência. Outro aspecto observado, nos trabalhos citados, é que o público-alvo mais atendido é o de ensino médio ou superior. Em nosso trabalho, buscamos estender as experiências também ao ensino fundamental, como será discutido a seguir.

\section{Método e Atividades}

As experiências aqui relatadas se desenvolveram no contexto de um projeto de extensão universitária, do qual participam bolsistas e voluntários, graduandos de cursos da área de Computação da Universidade Federal de Santa Maria, orientados por professores desta instituição. A equipe do projeto possui mais de 2 anos de experiência em contatos com escolas de ensino fundamental e médio, desenvolvendo atividades variadas com diferentes ferramentas (Scratch, Python, etc.). Esse público, em geral, nunca teve contato com fundamentos de computação antes de participarem das atividades do projeto. Em 2015, buscou-se ampliar a experiência, com o planejamento e execução de atividades com o App Inventor.

Pelas experiências anteriores, notou-se que os jogos para iniciantes oferecidos pelo projeto Hora do Código são eficazes para comunicar noções de programação visual, com encaixe de blocos. Por outro lado, essa abordagem oferece limitações ao potencial criativo dos alunos, pois baseia-se em tutoriais com objetivos em níveis crescentes de dificuldade. Com o MIT App Inventor, esperava-se contornar essa limitação, com a possibilidade de os alunos criarem seus próprios aplicativos, apoiados pela equipe do projeto (tutores).

Também pelas experiências anteriores, observou-se que a duração e frequência das atividades com os alunos é um aspecto crítico nesse tipo de projeto de extensão. Por um lado, atividades intensivas/frequentes são praticamente inviáveis, pois tanto nas escolas como na universidade os envolvidos possuem outras atividades. Por outro lado, atividades muito curtas e/ou espaçadas no tempo podem se tornar improdutivas e desestimulantes.

Considerando-se todas essas observações, decidiu-se planejar uma oficina com encontros semanais, somando de 4 a 6 horas no total. Essa oficina inicia com uma atividade da Hora do Código a ser realizada no período de uma hora e depois introduz o App Inventor, com o qual cada aluno cria um aplicativo na forma de um "Quiz" (jogo de 
perguntas e respostas), personalizado de acordo com seus interesses. Um tal aplicativo é apresentado num dos tutoriais do App Inventor ${ }^{3}$, servindo como referência para tutores do projeto, mas não diretamente para o público-alvo, por ser em inglês e usar uma abordagem que considerou-se "pesada" para o público das escolas.

Visto que o projeto visa executar as atividades de maneira informal e colaborativa, não apresenta-se uma introdução teórica à ferramenta, mas sim um primeiro contato através de uma visualização conjunta, com demonstrações e apresentação de slides, nas quais observam-se os principais recursos do App Inventor. Após, os participantes ficam livres para explorar a ferramenta, que por si só é auto-explicativa, pois cada recurso acompanha um botão que apresenta uma breve instrução sobre sua funcionalidade.

A atividade foi nomeada "Oficina de Criação de Aplicativos Móveis" e aplicada em duas ocasiões em 2015: com alunos de ensino médio na metade do ano e com alunos de ensino fundamental no final do ano.

\subsection{Primeira experiência}

Durante quatro semanas, em aproximadamente uma hora semanal, desenvolveu-se a experiência com uma turma de primeiro ano do Ensino Médio do Colégio Politécnico da UFSM, num laboratório de informática da própria escola. Para a escolha das datas e da turma mais apropriada, discutiu-se previamente com a coordenação da escola, que se mostrou interessada e disposta a ajudar. Esse apoio foi fundamental para que a oficina atingisse seus objetivos. De fato, em experiências anteriores, com menor envolvimento de professores ou coordenação das escolas, houve atividades com pouquíssimos ou nenhum participante.

De 31 alunos inscritos, 19 compareceram em pelo menos um encontro. Havia participantes de ambos os sexos. No primeiro encontro, apresentou-se o projeto, o cronograma de atividades e trabalhou-se a programação visual através da Hora do Código. A introdução ao MIT App Inventor ocorreu a partir do segundo encontro, quando lançou-se a proposta de criação de jogos personalizados de perguntas e respostas (Quizzes), cuja criação engloba mecanismos de raciocínio lógico similares aos mais utilizados para a programação em geral. Nesse ponto, forneceu-se um template (Figura 3) do jogo, com alguns blocos básicos mostrando como implementar uma pergunta/resposta no aplicativo, restando vários outros a serem inseridos ou configurados. Os encontros posteriores foram dedicados à customização e otimização dos aplicativos, e a execução desses no dispositivo móvel ou no emulador.

Na figura 3, é possível visualizar os blocos iniciais que foram disponibilizados aos alunos. Nesse template, estão contidos os blocos de inicialização de variáves (número e listas) para armazenar as perguntas e respostas (initialize), blocos de controle para executar ações ao iniciar a tela e ao clicar o botão de responder (when) e bloco de procedimento (Prox_Pergunta, para percorrer as listas). Nota-se também um bloco condicional (if) aninhado, para verificar se a resposta inserida está correta.

Durante os encontros, os alunos eram acompanhados quase individualmente pelos tutores, após uma breve explanação sobre os objetivos do encontro. Ou seja: a apresentação dos recursos da ferramenta foi a mais curta possível, abrindo espaço para

\footnotetext{
${ }^{3} \mathrm{http} / / /$ appinventor.mit.edu/explore/ai2/quizme.html
} 


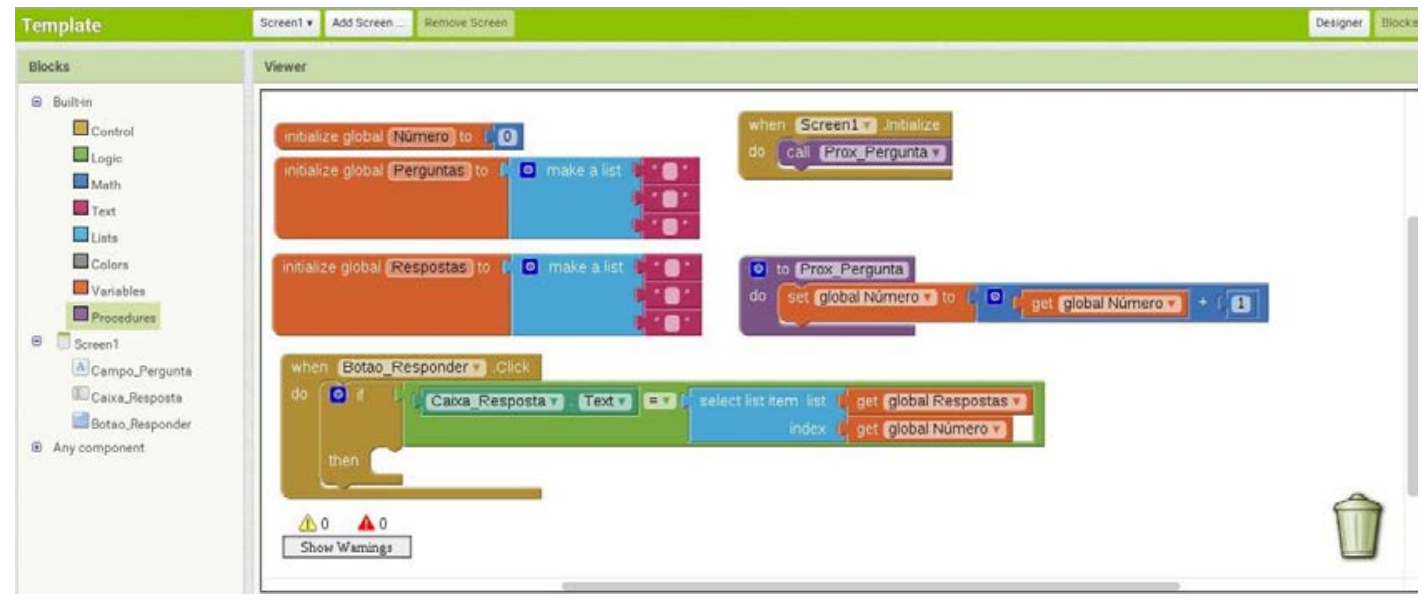

Figura 3. Tela "Blocos" com o template para a criação do aplicativo

que os alunos explorassem o MIT App Inventor na consecução dos objetivos propostos. Esses objetivos, por sua vez, eram moldáveis pelo interesse dos alunos. Por exemplo: o tema dos Quizzes poderia ser escolhido individualmente, assim como a quantidade de perguntas e o visual do aplicativo, entre outras características.

Entre os encontros, propôs-se um prêmio (uma camiseta do projeto) para os alunos que enviassem suas criações para o grupo de tutores. Dois alunos participaram, demonstrando autonomia em continuar a atividade em casa. No último encontro, com 9 alunos presentes, 6 deles enviaram seus aplicativos para a galeria do App Inventor (os demais não reservaram tempo para isso). Ao final desse encontro, todos os alunos preencheram um formulário avaliativo. A presença dos alunos no decorrer da oficina foi muito variada, portanto também enviou-se formulários àqueles que se inscreveram mas nunca compareceram e àqueles que compareceram em outros encontros, exceto no último. As questões do formulário visavam coletar impressões dos alunos sobre a oficina, identificando possíveis dificuldades e perspectivas de continuidade.

$\mathrm{Na}$ Figura 4, apresenta-se um exemplo de aplicativo criado por um aluno, com perguntas sobre matemática e geografia. Neste exemplo, é possível identificar os blocos utilizados e a interface do jogo no emulador.

\subsection{Segunda experiência}

Após novo planejamento, organizou-se outro cronograma para a segunda experiência. Dessa vez, a atividade se desenvolveu nas dependências da Universidade e foi aberta a alunos de diferentes escolas, com divulgação voltada principalmente ao público de Ensino Fundamental. A oficina ocorreu em dois sábados pela manhã, por ser um período livre para a maioria dos estudantes, com cerca de 3 horas por encontro, incluindo intervalo para lanche. De 16 alunos inscritos, 5 compareceram em ambos os encontros, sendo 2 meninas e 3 meninos. Esses alunos eram provenientes de 3 escolas e cursavam o $5^{\circ}$ ou $6^{\circ}$ ano do Ensino Fundamental.

A sequência do trabalho com o App Inventor foi semelhante à da experiência anterior, porém com uma melhoria: para a segunda oficina, desenvolveu-se um guia sobre o App Inventor, que foi entregue impresso a cada participante. Essa iniciativa apoiou-se num estudo [Xie et al. 2015] que sugere que usar tutoriais no primeiro contato com o App 


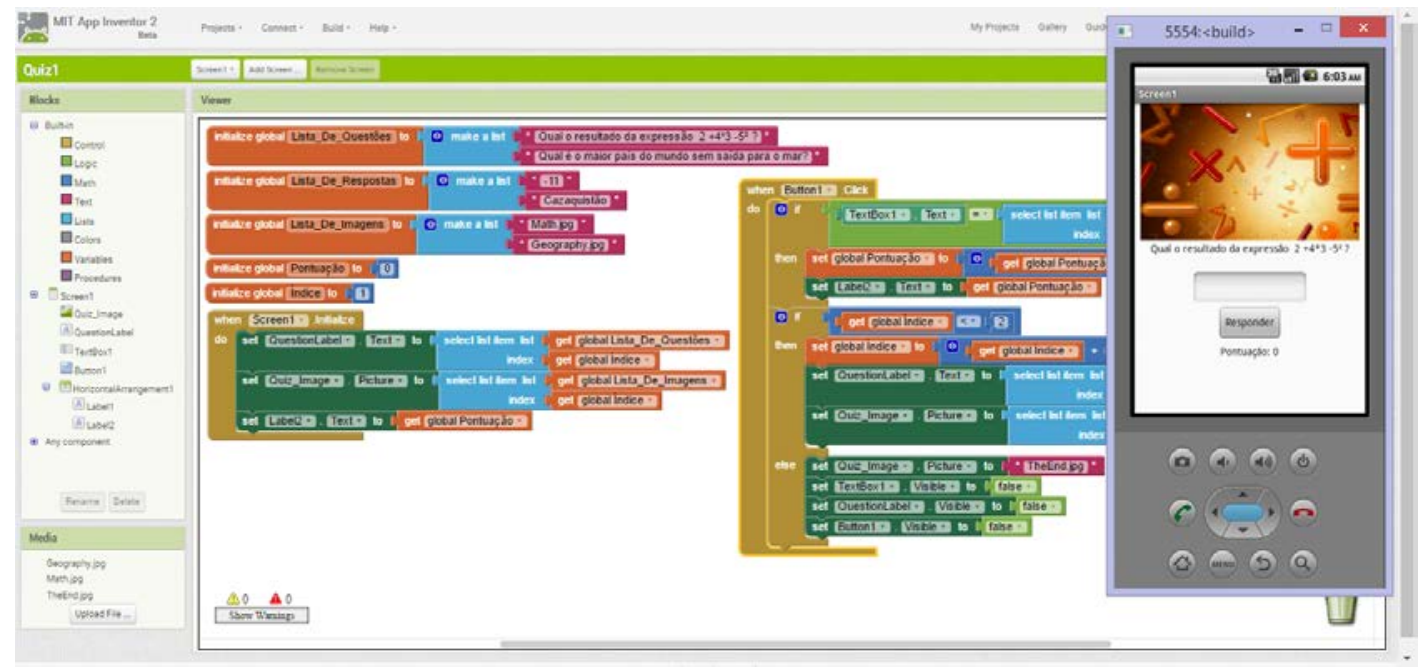

Figura 4. Exemplo de aplicativo produzido por um aluno

Inventor influencia na usabilidade percebida pelo usuário, no momento de implementar funcionalidades particulares. Esse guia foi organizado nas seguintes seções:

1. Primeira vez no MIT App Inventor?

2. Meu primeiro aplicativo

3. Aplicativo Quiz

4. Conhecendo melhor os blocos

5. Testando o aplicativo

6. Aprendendo a programar

7. Postando seu aplicativo na galeria

8. Mais informações

Entre os encontros, e mesmo após, manteve-se contato com os alunos via rede social, resolvendo dúvidas que surgiram quando esses tentaram utilizar o App Inventor em suas residências. A avaliação usou o mesmo formulário usado na primeira experiência.

\section{Resultados e Discussão}

De forma geral, as respostas dos alunos no formulário avaliativo revelaram boa aceitação da oficina, com níveis de dificuldade classificados como 3 ou 4, numa escala de 1 (mais baixo) a 5 (mais alto). Alguns alunos registraram comentários no formulário, que transcrevemos a seguir:

"Só acho que seria interessante uma continuação, onde pudéssemos nos aprofundar mais nos assuntos."

"Seria interessante novos encontros mais dinâmicos."

"A condução das atividades foi excelente. A quantidade de pessoas auxiliando colaborou bastante."

Embora os públicos nas duas experiências tivessem idades e níveis de escolaridade distintos, o desempenho dos alunos nas oficinas não revelou muita diferença. Em ambas as oficinas, alguns alunos progrediram mais rapidamente que outros, mas todos conseguiram atingir os objetivos propostos. Na segunda oficina, notou-se que a disponibilidade do guia impresso foi um grande facilitador. Uma observação que merece atenção 


\begin{tabular}{l|l|l|l}
\hline & Inscritos & $\begin{array}{l}\text { Maior número } \\
\text { de presenças }\end{array}$ & $\begin{array}{l}\text { Menor número } \\
\text { de presenças }\end{array}$ \\
\hline $\begin{array}{l}\text { Oficina 1: } \\
\text { Ensino Médio }\end{array}$ & 31 & 19 & 9 \\
\hline $\begin{array}{l}\text { Oficina 2: } \\
\text { Ensino Fundamental }\end{array}$ & 16 & 5 & 5 \\
\hline
\end{tabular}

Tabela 1. Números de inscritos e presenças nos encontros

diz respeito aos números de inscritos e presentes nos encontros, reunidos na tabela 5. Nessa tabela, colocou-se lado a lado o maior e o menor número de presenças em cada oficina, considerando-se todos os encontros.

O número de inscritos, nas 2 oficinas, foi maior do que o número de presenças. Esse comportamento já foi observado em todas as atividades anteriores do projeto de extensão e pode ser atribuído ao fato de que as atividades são gratuitas e o formulário de inscrição é disponível online, facilitando a inscrição de alunos que, depois, mudam de ideia.

Nota-se que os alunos do ensino fundamental, embora menos numerosos desde o início, estiveram presentes na totalidade dos encontros. $\mathrm{O}$ mesmo não se verificou com alunos do ensino médio, cujas presenças variaram mais. Mesmo considerando que a primeira oficina teve mais encontros, vale lembrar que a segunda ocorreu fora das escolas e aos sábados. Embora não tenhamos a pretensão de generalizar, essas observações são alinhadas com uma hipótese de que o público de ensino médio seja mais difícil de se manter estimulado com a proposta. Um dos motivos para isso pode ser a proximidade do ingresso no ensino superior, que se torna uma prioridade para muitos. De outro lado, os alunos de ensino fundamental, além de parecerem bastante atraídos pela proposta, são também influenciados pelos seus responsáveis, que os conduziram e, possivelmente, os estimularam a participar dos encontros.

Alguns obstáculos envolvendo a utilização do App Inventor foram observados ao longo dos encontros, pelos tutores, mesmo não sendo explicitamente apontados como dificuldades pelos alunos. Os mais frequentes foram:

- Interface em inglês: à época da realização das oficinas, o App Inventor não possuía interface em português, por isso foi usado o idioma inglês. Isso causou dispersão em alguns alunos, ao buscarem traduções paralelamente ao desenvolvimento dos aplicativos. Essa dificuldade também foi observada em outro trabalho [Ramos et al. 2015]. Atualmente, o App Inventor já possui interface em português, mas notou-se que a primeira caixa de diálogo da ferramenta continua em inglês.

- Teste dos aplicativos: conforme já mencionado, o App Inventor inclui um emulador de dispositivo móvel, no qual os aplicativos podem ser testados. Outra opção é baixar o aplicativo no próprio celular. Em ambos os casos, notou-se dificuldades: o emulador muitas vezes interrompe a comunicação com a ferramenta ou leva um tempo considerável para atualizar a visualização, o que causa muitas dúvidas aos alunos; já a instalação no celular não é feita como os alunos estão habituados (via Google Play), por isso também causa dúvidas. 
- Blocos condicionais: a abordagem utilizada não partiu de uma exposição detalhada dos blocos, mas mesmo assim a lógica do Quiz e muitos blocos foram rapidamente compreendidos pelos alunos. No entanto, blocos condicionais geraram muitas dúvidas não somente pela lógica, mas pela necessidade de muitos encaixes e configurações menos intuitivas.

É importante ressaltar que a presença dos tutores foi fundamental nas oficinas. Pela abordagem utilizada, as crianças e jovens foram estimulados a explorar o App Inventor e solicitar ajuda quando tivessem dificuldades. Na prática, cada tutor assistiu um ou dois alunos, acompanhando as criações durante os encontros. Essa abordagem inviabiliza oficinas com grande número de participantes, que necessitaria de muitos tutores. Felizmente, nossa experiência com esse tipo de ação extensionista tem mostrado que os graduandos são atraídos pela possibilidade de interagir com o público externo e, mais do que isso, contribuir para disseminar fundamentos da área. Assim, pudemos contar com 10 graduandos voluntários atuando como tutores em diferentes ocasiões.

\section{Considerações Finais}

As experiências realizadas aproximaram alunos de ensino médio e fundamental a uma possibilidade que até então lhes parecia distante: eles/elas foram capazes de criar aplicativos para seus celulares. Assim como outras intervenções extensionistas relatadas na literatura, estas experiências não só aproximaram a Universidade da comunidade, mas também estimularam o interesse de crianças e jovens em aprofundar seus conhecimentos na área de Computação. Para graduandos, a experiência também revelou-se estimulante, atraindo voluntários e não somente bolsistas.

Visando facilitar a replicação deste tipo de experiência, o material desenvolvido e utilizado nas oficinas encontra-se disponível em: facebook.com/CompClubUFSM. Sugere-se, em ações futuras, levar em consideração as seguintes lições aprendidas:

- Alunos de ensino fundamental ficam tão à vontade quanto os do ensino médio frente ao App Inventor. Assim, caso se tenha que priorizar uma ou outra faixa etária beneficiada com a experiência, sugere-se iniciar com os de ensino fundamental, por ser um público com menos preocupações (ingresso no ensino superior) e que, potencialmente, seguirá mais facilmente participando de novas ações extensionistas que visem aprofundar o conhecimento de fundamentos de Computação.

- Usar material impresso durante as atividades, embora tenha um custo adicional, é uma estratégia vantajosa para estimular a autonomia dos alunos. Nota-se que, disponibilizando esse material fisicamente (e não somente online), os alunos passam a consultá-lo antes de solicitar ajuda de um tutor.

- Embora o App Inventor seja uma ferramenta com recursos intuitivos, ele impõe também alguns obstáculos a usuários iniciantes. Nesses pontos, mesmo que exista documentação explicando os blocos e outros componentes, é fundamental o apoio de um tutor, pois do contrário os alunos podem facilmente se desestimular.

\section{Referências}

de Cerqueira Cardoso, J. P. P., Morais, L. C., Rodrigues, C. A., e Bittencourt, R. A. (2015). Introduzindo programação de computadores e PBL com python e App Inventor. In Anais do XLIII Congresso Brasileiro de Educação em Engenharia - (COBENGE), pages 1 - 10, Feira de Santana, BA. 
Duda, R., Zontini, D. D., de Carvalho Rutz da Silva, S., e Grossi, L. (2014). Elaboração de aplicativos para Android com o uso do App Inventor: uma experiência no instituto federal do paraná - câmpus irati. In Anais do IV SINECT - Simpósio Nacional de Ensino de Ciência e Tecnologia, pages 1 - 12, Ponta Grossa, PR.

Finizola, A. B., Raposo, E. H. S., Pereira, M. B. P. N., Gomes, W. S., de Araújo, A. L. S. O., e Souza, F. V. C. (2014). O ensino de programação para dispositivos móveis utilizando o MIT-App Inventor com alunos do ensino médio. In Anais do Workshop sobre Informática na Escola - (WIE), pages 337 - 341.

Fronza, I., El Ioini, N., e Corral, L. (2015). Students want to create apps: Leveraging computational thinking to teach mobile software development. In Proceedings of the 16th Annual Conference on Information Technology Education, SIGITE '15, pages 21-26, New York, NY, USA. ACM.

Gardner, H. e Davis, K. (2013). The App Generation: How Today's Youth Navigate Identity, Intimacy, and Imagination in a Digital World. Yale University Press.

Gomes, T. C. S. e de Melo, J. C. B. (2013a). Lógica de programação: Iniciação lúdica com app inventor for android. Revista da Escola Regional de Informática de Pernambuco, pages $155-161$.

Gomes, T. C. S. e de Melo, J. C. B. (2013b). O pensamento computacional no ensino médio: Uma abordagem blended learning. In Anais do Workshop sobre Educação em Computação - (WEI), pages $1-10$.

Hulme, A. K. e Traxler, J. (2005). Mobile Learning: A Handbook for Educators and Trainers. Routledge.

Khalaf, S. (2015). Mobile addicts multiply across the globe. Flurry Analytics.

Ramos, N., Freitas, C., Avila, S., Costa, P. D. P., Testoni, V., e Borin, J. F. (2015). Ensino de programação para alunas de ensino médio: Relato de uma experiência. In Anais do Workshop sobre Educação em Computação - (WEI), pages 1 - 10.

Rideout, V. J., Foehr, U. G., e Roberts, D. F. (2010). Generation M2: Media in the Lives of 8- to 18-Year-Olds. Technical report, Henry J. Kaiser Family Foundation.

Wolber, D. (2011). App inventor and real-world motivation. In Proceedings of the 42Nd ACM Technical Symposium on Computer Science Education, SIGCSE '11, pages 601606, New York, NY, USA. ACM.

Xie, B., Shabir, I., e Abelson, H. (2015). Measuring the usability and capability of app inventor to create mobile applications. In Proceedings of the 3rd International Workshop on Programming for Mobile and Touch, PROMOTO 2015, pages 1-8, New York, NY, USA. ACM. 